\title{
ACUTE GASTROENTERITIS WITH CLOSTRIDIUM DIFFICILE IN A SMALL CHILD WITH HEREDITARY LACTOSE INTOLERANCE
}

\author{
Maria Oana Marginean', Raluca Damian², Andreea Dinca ${ }^{3}$, \\ Cristina Oana Marginean ${ }^{1,3}$, Lorena Elena Melit ${ }^{1,3}$ \\ ${ }^{1}$ University of Medicine and Pharmacy, Targu-Mures \\ ${ }^{2}$ Neonatology Clinic I, Targu-Mures \\ ${ }^{3}$ Pediatrics Clinic I, Targu-Mures
}

\begin{abstract}
Acute gastroenteritis with Clostridium difficile is insufficiently studied in the specialty literature due to its small incidence at this age, with increased rates of morbidity and mortality. We present the case of a 1 year and 10 month-old child, diagnosed with hereditary lactose intolerance in the neonatal period, admitted in the Pediatrics Clinic I, Targu-Mures, for vomiting, loss of appetite, decreased consistency stools, with a bed smell, with bacterial infection signs in the blood tests and slowly favorable evolution under treatment with cephalosporin. The patient is readmitted after 1 day from discharge with the reappearance of bad smell stools and loss of appetite, with unfavorable evolution after administering a $3^{\text {rd }}$ generation cephalosporin, thus we raise the suspicion of infection with Clostridium difficile, confirmed by the identification of toxins in the stools, with favorable evolution under treatment for anaerobe germs (metronidazole). The particularity of the case consists in the development of a gastroenteritis with Clostridium difficile in a small child (1 year and 10 months), known with retardation in the neuro-psychomotor and language development, repeated episodes of hypoglycemia, with hereditary lactose intolerance which represented a challenge in establishing the diagnosis.
\end{abstract}

Keywords: Clostridium difficile, child, gastroenteritis, hereditary lactose intolerance

\section{INTRODUCTION}

Acute gastroenteritis is defined by the modification of the stools consistency associated or not with increase in stools frequency, in febrile context or not, with or without vomiting. Acute diarrheic disease is one of the most frequent in the pediatric age patients. The etiology of this pathology is in most of the cases viral, but the bacterial and parasite etiology are not rare. Even though the infection with Clostridium difficile is perhaps one of the most frequent nosocomial infections relatively frequently encountered in the adult's pathology, in pediatric age patients, few data are available in the specialty literature regarding this infection. The incidence of this infection increased in the last decades, most probably due to the wide use of antibiotics. Thus, in the last decades, it is described in some areas of the globe an increase of the annual incidence among children of approximately 55\% (1). Gastroenteritis with Clostridium difficile can affect any age, being most frequently a nosocomial bacteria, even though the community-acquired infection is not excluded. In most of the cases it appears in susceptible patients. Thus, among the most frequent risk factors are: associated comorbidities, use of antibiotics, invasive mechanic ventilation, feeding by nasogastric tube and immunosuppression (2). The diagnosis of this infection is established by the identification of $\mathrm{A} / \mathrm{B}$ toxins in the stools, or in rare cases, in which the clinical picture suggest this infection, with discordant results regarding the toxin identification, we can use also the genetic method polymerase-chain reaction (PCR) (3). The treatment included agents with anaerobe spectrum, such as metronidazole or vancomycin in case of children. The complications of this bacterial infections are very severe in most of the case, leading to the increase of morbidity and mortality rates among general population, being associated in case of children with a 6 folded risk of death during admission (4).

Corresponding author:

Oana Marginean, University of Medicine and Pharmacy, 38 Gh. Marinescu Street, Targu-Mures

E-mail: marginean.oana@gmail.com 


\section{CASE PRESENTATION}

We present the case of a female small child, 1 year and 10 months old, known since the age of 3 weeks with hereditary lactose intolerance, admitted in the Pediatrics Clinic I, Targu- Mures, with vomiting, loss of appetite, serous rhinorrhea and decreased consistency stools, with bad smell. From the familial history, we discovered that the mother is diagnosed with lactose intolerance. The physiological personal history pointed out that the patient is the second child, from a pathological pregnancy (at the 19-20 gestational weeks, she presented right hydrothorax resorbed spontaneously, negative amniocentesis), born at term, by cesarean section, with a birth weight of $3,830 \mathrm{~g}$ and APGAR score 8/1 minute and 9/5 minutes, fed with free-lactose formula since the age of 3 weeks. The pathological personal history pointed out neonatal sepsis, hereditary lactose intolerance, retardation in the neuro-psychomotor and language development. We mention that in the first 6 months of life, the patient presented multiple episodes of hypoglycemia, the glycemic levels being very difficult to maintain in normal limits until the moment of diversification. The onset of the actual disease was 6 before the admission, with vomiting, loss of appetite, serous rhinorrhea and decreased consistency stools, reasons for which the mother took her to the general practitioner who recommends antibiotic and symptomatic treatment, but with unfavorable evolution, therefore she was admitted in our clinic for investigations and specialty treatment.

The clinical exam at the moment of admission revealed the following pathological elements: slightly influenced general status, dry skin, diminished cutaneous turgor, discreetly hyperemic pharynx, serous rhinorrhea, slightly diffuse painful abdomen at palpation, present bowel movements, decreased consistency stools, without meningeal irritation signs, retardation in the neuro-psychomotor and language development, W: $11.5 \mathrm{~kg}$. The $\mathrm{CBC}$ count pointed out a slightly increased number of leukocytes (Leu $13640 / \mu \mathrm{L}$ ) with neutrophilia (Neu 60.4\%) with negative acute phase reactants and a glycemic level of $53.4 \mathrm{mg} / \mathrm{dL}$. We also performed stool culture, parasitological exam and Rotavirus test from the stools, but all were negative. Thus, we initiated symptomatic treatment with Metoclopramid, Controloc, Debridat and Smecta, but in the second day of admission, she developed fever $\left(39^{\circ} \mathrm{C}\right)$ and an increase of stool frequency, with bad smell, and increase of the acute phase reactants (PCR $21.91 \mathrm{mg} / \mathrm{L}$ ), reasons for which we decided to initiate antibiotic treatment (Axetine). The abdominal ultrasound revealed abdominal bloating. The evolution was slowly favorable, with the remission of the stools and fever, but with the maintenance of quirky appetite. After approximately 5 days of admission, the patient was discharged in relatively good general status, without presenting vomiting or diarrhea, with the recommendations of continuing the antibiotic treatment at home for 2 days associated with probiotics. After approximately 24 hours from discharge, the mother presented again with the child due to the fact that the loss of appetite reappeared, refusing to consume per mouth, therefore se is readmitted in our clinic. After the admission, she associates again decreased consistency stools with bad smell, reason for which we decided to change the antibiotic regimen introducing a $3^{\text {rd }}$ generation cephalosporin (Ceftamil). The blood tests pointed out leukocytosis (Leu $14,970 / \mu \mathrm{L}$ ) with neutrophilia (Neu $8,700 / \mu \mathrm{L}$ ), and positive acute phase reactants (PCR $24.3 \mathrm{mg} / \mathrm{L}$ ). During the admission day, we performed again stool culture and Rotavirus test from the stools, but the results remained negative. After approximately 3 days from admission, the stools continued to have decreased consistency, bad small and mucus, therefore we raised the suspicion of infection with Clostridium difficile confirmed by the identification of toxins from the stools. Thus, we introduced treatment with metronidazole, $3 \times 80 \mathrm{mg} /$ day by mouth, for 5 days, with favorable evolution. We also mention that the diet management represented a particular aspect in this case due to the hereditary lactose intolerance and to the history of repeated episodes of hypoglycemia, therefore during the evolution of the disease, the patient needed supplementation with glucose by vein with frequent monitoring of blood glucose, before every meal and in case of suggestive clinical symptomatology. Once, she regained the appetite and the stools had a normal aspect, the perfusion with glucose was ceased, but we continued to monitor the glycemic values during admission. The recommendations at discharge regarding the diet, were to avoid the concentrated sweet food, to use as much as possible aliments rich in carbohydrates, with successive release of glucose in order to maintain constant values of blood glucose (rise, meat, potatoes, cereals etc.), without fresh fruit or vegetables for approximately one month, monitoring the glycaemia at least once a week. The particularity of the case consists in the development of a gastroenteritis with Clostridium difficile in a small child (1 year and 10 months), known with retardation in the neuro-psychomotor 
and language development, repeated episodes of hypoglycemia, with hereditary lactose intolerance which represented a challenge in establishing the diagnosis

\section{DISCUSSIONS}

The incidence of Clostridium difficile infection increased dramatically in the last decades accordingly to several studies performed in the United States of America (5-7). This fact seems to be a result of the wide use of antibiotics. Regarding the class of antibiotics most frequently associated with Clostridium difficile infection, Fisher underlined in his study performed on 8,268 children diagnosed with acute lymphoblastic leukemia the following: beta-lactam antibiotics take the first place, while the use of cefepime or ceftazidime are independently of this infection (8). In case of our patient, the initial antibiotic used was cefuroxime (Axetine), and afterwards ceftamil, none of them belonging to the beta-lactam class, reason for which we tend to believe that our patient was an asymptomatic carrier of this anaerobe bacterium, fact described also by the specialty literature (9). Even though the infection with Clostridium difficile was typically known as a nosocomial infection, it seems that the incidence of the community-acquired cases is increasing, therefore in a study performed on 684 cases of infection with Clostridium difficile, 304 were community-acquired, 338 were nosocomial ones, and 42 undetermined ones (10). In the west of Romania, the incidence per hospital is approximately $20.47 / 15.7$ at 1000 patients discharged in $2013 / 2014$, with $0.62 \%$ under the age of 20 years (11). Regarding the risk factors in the studied group, it seems that more than one half of the patients who developed this infection received antibiotics, and in comparison with the study mentioned above, most of them received cephalosporins and fluoroquinolones $(31.03 \%$ and $28.96 \%$, respectively) (11). The risk factors predictable for unfavorable evolution identified in this study were the age, the number of leukocytes over 16,000 de cells $/ \mu \mathrm{L}$, hypoalbuminemia under $3.5 \mathrm{~g} / \mathrm{dL}$, fever over $37.5^{\circ} \mathrm{C}$, chronic lung disease or moderate/severe liver disease, chemotherapy, parenteral nutrition, continuation of the antibiotic treatment after the onset of diarrhea (others than those for Clostridium difficile), the number of days of antibiotic administration and the number of antibiotics (11). Still, the children develop more frequently community-acquired infection with Clostridium difficile than the adults (12), probably also due to the fact that they can be reservoirs of the infection, being asymptomatic carriers. The treatment is the same in both, adults and children, but it seems that the pediatric age patients respond better to metronidazole, similarly to our case, while adults respond better to vancomycin (12). The consequences of acute gastroenteritis with Clostridium difficile at pediatric age include: the prolongation of the hospitalization length, increased costs related to the treatment, increased rates of colectomy comparatively with the adults, but also re-admissions due to the infection recurrence or unfavorable evolution (12), similarly to our case. The treatment of the infection with Clostridium difficile consists in metronidazole (first choice) or vancomycin associated with rehydration measures (12). Regarding the prevention of this infection is children, it is based mainly on the careful use of antibiotics, but also on the administration of probiotics, which seem to reduce up to $60 \%$ the infection rate with this anaerobe germ (13). Despite this facts, a study performed in the United Stated identified 29,000 deaths in 2011 due to this infection (14).

\section{CONCLUSIONS}

Acute gastroenteritis with Clostridium difficile is a disease with severe complications in pediatric age. Its typical nosocomial character is less and less specific, with the increase of the community-acquired infections, fact that should aware the clinicians and to determine them to be more responsible regarding the ambulatory prescription of antibiotics. The alarming increase in incidence of Clostridium difficile infection among children in the last decades should determine the pediatrician to suspect this infection in order to establish as early as possible the diagnosis and to administer an adequate therapy for preventing the complications.

\section{REFERENCES}

1. de Blank P., Zaoutis T., Fischer B. et al. Trends in Clostridium difficile infection and risk factors for hospital acquisition of Clostridium difficile among children with cancer. J Pediatr 2013; 163:699-705.
2. Maccioni A., Cerda J., Terrazas C., Abarca K. Clinic and epidemiologic description of Clostridium difficile infection in a pediatric population. Rev Chilena Infectol 2015; 32(5):523-9. 
3. Gonzalés-Abad M.J., Alonso-Sanz M. New methodological advances: algorithm proposal for management of Clostridium difficile infection. Rev Esp Quimioter 2015; 28(3):157-9.

4. Sammons J.S., Localio R., Xiao R. et al. Clostridium difficile infection is associated with increased risk of death and prolonged hospitalization in children. Clin Infect Dis 2013; 57:1-8.

5. Kim J., Smathers S.A., Prasad P. et al. Epidemiological features of Clostridium difficile-associated disease among inpatients at children's hospitals in the United States 2001-2006. Pediatrics 2008; 122:1266-70

6. Zilberberg M.D., Tillotson G.S., McDonald C. Clostridium difficile infections among hospitalized children, United States, 1997-2006. Emerg Infect Dis 2010; 16:604-9.

7. Nylund C.M., Goudie A., Garza J.M. et al. Clostridium difficile infection in hospitalized children in the United States. Arch Pediatr Adolesc Med 2011; 165:451-7.

8. Fisher B.T., Sammons J.S., Li Y. et al. Variation in Risk of HospitalOnset Clostridium difficile Infection Across $\beta$-Lactam Antibiotics in Children with New-Onset Acute Lymphoblastic Leukemia. Pediatr Infect Dis Soc 2014; 3(4):329-35.
9. Tang P., Roscoe M., Richardson S.E. Limited clinical utility of Clostridium difficile toxin testing in infants in a pediatric hospital. Diagn Microbiol Infect Dis 2005; 52(2):91-4.

10. Kuntz J.L., Crischilles E.A., Pendergast J.F., Herwaldt L.A., Polgreen P.M. Incidence of and risk factors for community-associated Clostridium difficile infection: A nested case-control study. BMC Infect Dis 2011; 11:194.

11. Laza R., Jurac R., Crisan A., Lăzureanu V. et al. Clostridium difficile in western Romania: unfavourable outcome predictors in a hospital for infectious diseases. BMC Infect Dis 2015; 15:141.

12. McFarland L.V., Ozen M., Dinleyici E.C., Goh S. Comparison of pediatric and adult antibiotic-associated diarrhea and Clostridium difficile infections. World J Gastroenterol 2016; 22(11):3078-104.

13. Johnston B.C., Ma S.S., Goldenberg J.Z., Thorlund K., Vandvik P.O., Loeb M., Guyatt G.H. Probiotics for the prevention of Clostridium difficile-associated diarrhea: a systematic review and meta-analysis. Ann Intern Med 2012; 157:878-88.

14. Lessa F.C., Bamberrg W.M., Beldavs Z.G. et al. Burden of Clostridium difficile infection in the United States. N Engl J Med 2015; 372:825-34. 